\title{
Detailed Damage Assessment after the Haiti Earthquake
}

\author{
Danielle HOJA, Thomas KRAUß and Peter REINARTZ \\ DLR · Remote Sensing Technology Institute · Oberpfaffenhofen · 82234 Weßling · Germany \\ Tel.: +49 (0) $8153282725 \cdot$ danielle.hoja@dlr.de
}

\section{Keywords: Damage assessment, change detection, very high resolution (VHR)}

\begin{abstract}
Summary: In the post crisis phase a much more detailed analysis can be done with higher accuracy and less pressure of time compared to the general situation assessment of the rapid mapping process directly after the crisis. In this investigation the analysis is concentrated on the urban area of the capital town Port-au-Prince. In order to develop a service for detailed damage assessment, methods of (semi-)automatic change detection are used and compared, since up to now, damage information was mainly derived by visual interpretation. Any improvement in terms of accuracy and speed of analysis is of relevance to users in this context. The results of the different change detection algorithms achieved using the Haiti datasets are compared to each other and also to the database of the Haiti Action Plan for Reconstruction and Development (PDNA). The results are very promising although further improvements have to be made..
\end{abstract}

\section{Introduction}

Directly after a crisis or disaster an important part of information generation for the response and relief effort is the production of crisis maps (rapid mapping) for humanitarian relief organisations. For immediate aid and the general situation assessment fast information is needed about damages to infrastructure and affected areas. This analysis is expected as fast as possible and even important if not exact in all details..

A more detailed analysis of the damage situation can be done in the post crisis phase with much higher accuracy, but with less pressure of time. Users are interested in a detailed damage assessment to support the estimation of losses and funds needed for reconstruction. The information may be used during post-conflict needs assessments, in donor conferences or in bilateral/multilateral negotiations. Some time after a crisis in the rehabilitation and reconstruction phase, the development of the infrastructure rebuilding can be monitored by the same methodology. Especially a supervision of an appropriate utilisation of the allocated funds is of interest to the donators.

All these services, i.e. rapid mapping directly following a crisis or catastrophic event, longterm damage assessment and reconstruction monitoring, are developed in the EU project GMES Services for Management of Operations, Situation Awareness and Intelligence for Regional Crisis (G-MOSAIC) and applied to data sets acquired after crisis situations as the Haiti earthquake. They are all implemented in so-called "Service Chains" describing the data flow and processing done by several partners in the project.

On January 12, 2010 a severe earthquake took place at the coast of Haiti with a magnitude of 7.0 causing major damage. The epicentre was registred about $10 \mathrm{~km}$ deep and $16 \mathrm{~km}$ southwest of the capital city Port-au-Prince. In the city a large number of buildings such as the palace, government and UN-buildings, embassies and hospitals were affected or destroyed. Telecommunication as well as electricity and water supply failed. In this article we show first results of the long-term damage assessment service chain in the context of the Haiti earthquake.

\section{Data sets and Preprocessing}

The analysis is concentrated on the urban area of the capital town Port-au-Prince. Different datasets are available for the analysis, mainly scenes of the very high resolution (VHR) GeoEye-1 satellite providing data with $50 \mathrm{~cm}$ ground sampling distance (GSD) and stereo capabilities. Three scenes were acquired: October 2009 (before crisis, no stereo), January 2010 (four days after the earthquake, stereo) and August 2010 (after crisis, stereo). The 
rapid mapping results collected in the database Post Disaster Needs Assessment and Recovery Framework (PDNA) are used for comparison. Also the airborne data used to receive these PDNA database are available for the study. Additionally, a manual change detection was carried out directly with the data sets for a first evaluation of the algorithms taking into account the state of change directly at the time of acquisition of the satellite images.

An important requirement for automatic change detection is a very good co-registration of pre- and post-disaster scenes. One of the scenes to be analysed is defined as reference scene and all other images are co-registered onto this scene. The automatic processing chain developed at DLR combines the orthorectification with the co-registration, so only one resampling step is necessary (Müller et al., 2010). After an automatic image matching with respect to the reference scene, the information for the direct georeferencing (attitude data or RPC) is adjusted using these matched points. With this information and a digital surface model (DSM) the scene is orthorectified providing co-registered results with better than $1 / 2$ pixel accuracy. Additionally a pansharpening is applied to the data sets taking into account the high spatial resolution of the panchromatic image as well as the radiometric information of the multispectral data. Fig. 1 shows a subset of the co-registrated and pansharpend images of the Haiti dataset.
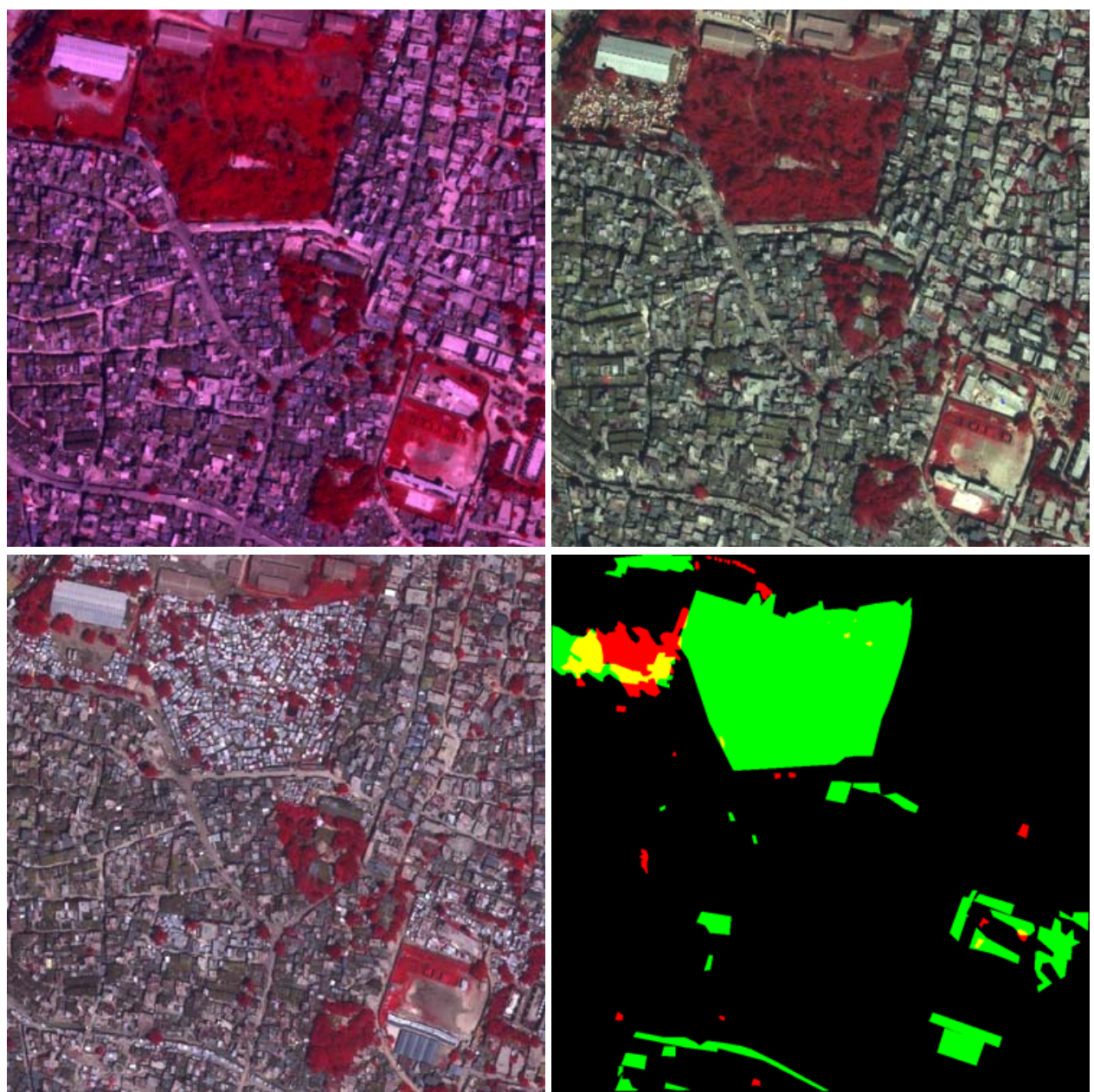

Fig. 1: Subset $(500 \mathrm{~m} \times 500 \mathrm{~m}$ ) of Port-au-Prince, Haiti showing the development of a refugee camp after the earthquake on 2010/01/12. (Top left) GeoEye, 2009/10/01 - PRE; (Top right) GeoEye, 2010/01/16 - PO1; (Bottom left) GeoEye 2010/08/18 - PO2; (Bottom right) Manual measured changes between $\mathrm{PRE} \rightarrow \mathrm{PO} 1$ and between $\mathrm{PO} 1 \rightarrow \mathrm{PO} 2$ (areas changed in both time frames are yellow)

As two of the scenes were available as stereo scenes, a new approach was tested to orthorectify the images using the digital surface model generated by the stero pairs. This 
leads to more accurate results compared to the orthorectification using other DSM (e.g. SRTM) not only due to the better resolution but also due to the better co-registration of images and DSM (d'Angelo et al., 2009). Therefore, at first digital surface models are derived by dense stereo matching and forward intersection and subsequent interpolation into a regular grid. Orthorectified images are produced in the second step using the affine RPC correction and the generated DSM.

The resulting DSM contains a a small amount of blunders, e.g. due to matching errors in regions with sparse texture. These blunders cause some distortions in the resulting orthoimage, that are responsible for some errors in the change detection. In future, these blunders have to be removed and the holes in areas where the matching failed or outliers were removed have to be filled, e.g. with SRTM data using the delta surface fill method (Grohman et al., 2006).

\section{Change Detection Methods - Application and Results}

Both service chains of long-term damage assessment and reconstruction monitoring use the methods of change detection (CD). Up to now, damage information was mainly derived by visual interpretation of pre- and post-disaster satellite data. Any improvement in terms of accuracy and speed of analysis using the methods developed within the G-MOSAIC project is of relevance to users in this context.

After the co-registration, different change detection methods like differencing are applied to the datasets. In a second step more complex methods, e.g. multivariate alteration detection (MAD) or 3D change detection are used. The results gained automatically are analysed manually for the high accuracy requested by the user. However, such a semiautomatic service chain is still faster than a complete manual analysis.

\subsection{Basic Change Detection: Image Differencing}

For image differencing, an additional pre-processing step is necessary. Due to some acquisition parameters such as different acquisition dates and view angles of the sensor, there are changes that are not of interest to the user (virtual changes). The radiometric correction step aims at reducing these differences of the two temporal images caused by the variation in light and atmospheric conditions at the two acquisition dates (Bovolo \& Bruzzone, 2007). Here histogram normalization is used for image differencing. The histogram of the pre-event image (October 2009) is taken as reference and the histograms of the two post-event images are adjusted to it.

Next, the two co-registered images are simply subtracted pixel by pixel. Image differencing is mathematically the easiest and the most widely used automatic change detection approach that has been applied in various geographical environments (Singh, 1989). Ideally, the value zero represents areas of no change while positive or negative values stand for changed areas in the difference image. Since many factors such as inaccurate coregistration can lead to virtual changes, pixels in no change areas do not necessarily have zero values. The critical step of image differencing is deciding where to place the threshold boundaries between change and no-change (Singh, 1989).

The selection of the correct threshold is often done using some in-situ information or the result of a manual analysis. Since the algorithm is planned as completely automatic, the threshold selection is based on statistical values. Pixels in the range of "mean +/- standard deviation" are considered "no change", pixels outside this range are considered "change". These values were received in a similar study (Liang, 2010; Liang et al., 2011). For multispectral images, different ways exist to deal with the single bands. In our approach, all steps are carried out for each band separately. Only after the thresholding of the image differencing result, the results obtained by single bands are combined. Best results were received taking into account only pixels indicating a change in at least three bands.

Fig. 2 shows the examplary result for the changes between the two post-disaster images (four days and seven months after the earthquake). The upper left image shows the difference for band 3 . The histogram (top right) shows the result for all 4 bands. The values are changed into 8bit (by adding 256 and dividing by 2). Mean value for band 3 is 127.8 
(similar for all bands), the standard deviation is 17.3 (smallest value, up to 27.9 for band 2). The lower left image shows the resulting change detection map (binary image). After the removal of pixels indicating change only in one or two bands, some morphological operators were also applied (opening, closing) to remove spurious pixels. In the lower right corner the image is overlayed with the manual measurement.

The colours in this overlay image indicate the correctness of the automatic algorithm in comparison to the manual measurement. Green areas indicate changes in both the automatic derived binary map as well as in the manual measurement, whereas black indicates "no change" in both change maps. Blue colour indicates areas, which have changed according to the manual measurement but were not detected by the automatic algorithm. As can easily be seen, there are no solely blue areas, blue areas occur only in combination with green areas. So there are at least some changes nearby, and a manual verification of the automatic measurements will finish with the correct result. Additionally, it can be seen, that the manual measurement marked large areas of change (green and blue together), e.g. the complete refugee camp as one changed area. As can be seen in Fig. 1, there are still some trees inside this area, which of course do not appear as change in the automatic algorithm. Finally, red colour indicates areas defined by image differencing as change, but not in the manual measurement. These areas are mostly changes not of interest (i.e. vegetation changes) and have to be removed in a following verification step. However, the areas to be analysed (red and green) are significantly smaller than the complete image, which has been analysed manually so far.
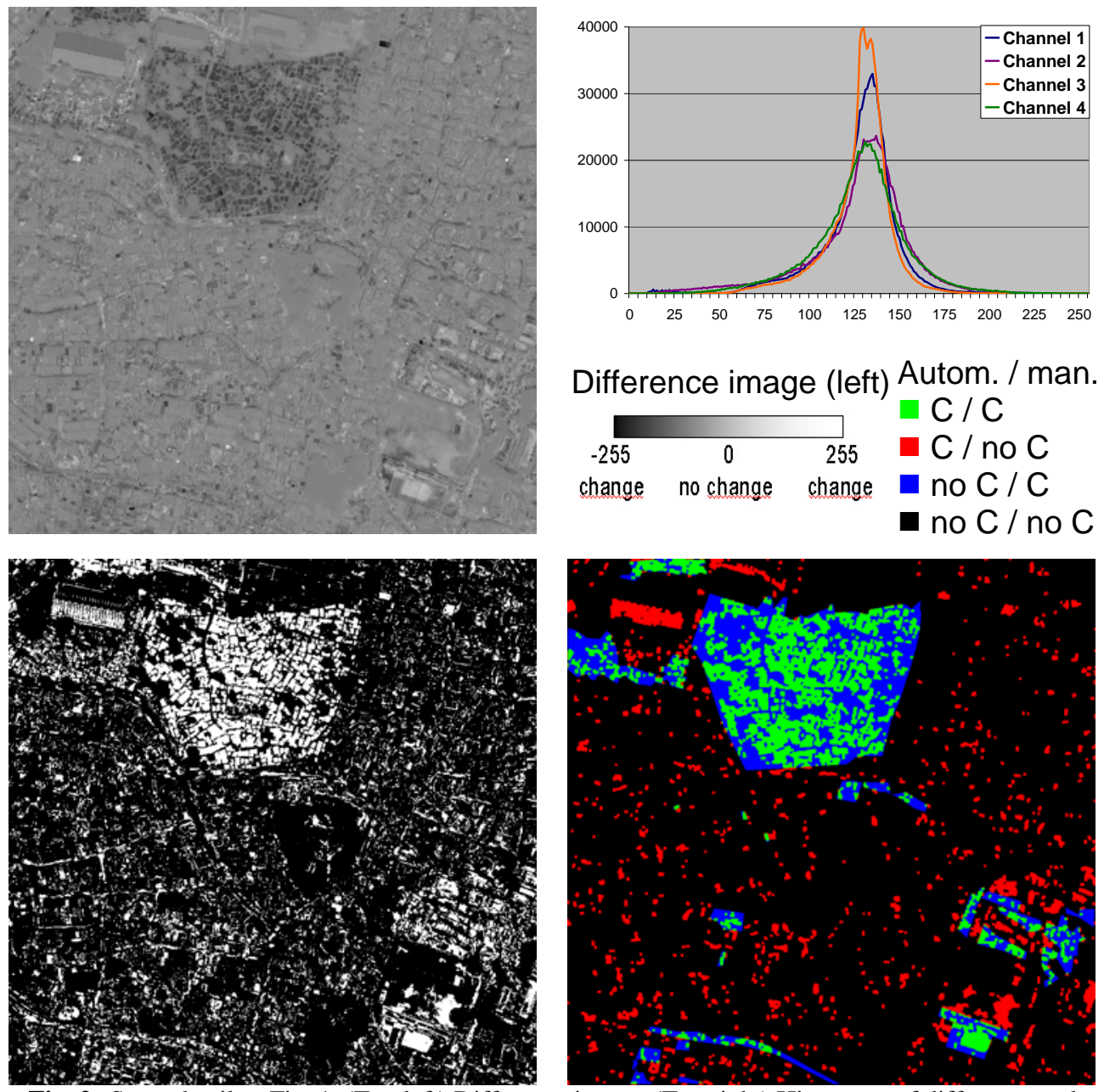

Fig. 2: Same detail as Fig. 1. (Top left) Difference image; (Top right) Histogram of difference and legends; (Bottom left) Resulting binary change detection map, White = change; (Bottom right) Overlay of difference binary map (“Autom.”) and manual measurement (“man.”; C = Change) 


\subsection{Multivariate Alteration Detection (MAD)}

To avoid radiometric correction as well as band selection, another promising differencebased change detection method is iteratively reweighted multivariate alteration detection (IR-MAD). MAD analyses multispectral data by utilizing all the bands simultaneously. The method is based on an established multivariate statistical technique: canonical correlation analysis (CCA). As CCA finds a linear combination of the original multispectral data ordered by decreasing correlation between pairs, MAD transformation determines the difference between linear combinations of the original multispectral bands ordered by variance. A detailed analysis can be found in (Nielsen, 2007).

IR-MAD calculates original MAD variates firstly, and then in the following iterations, puts large weights on the observations which show little change. This is achieved through calculating a measure of no change based on the sum of squared, standardized MAD variates in each iteration, little change means that the sum of squared, standardized MAD variates is small (Nielsen, 2007). The iterations are continued until the largest absolute change in the canonical correlations reaches a predefined value, e.g. $10^{-3}$.

Additionally to the four multispectral bands, the input image to the MAD algorithm is combined with some texture information. It is based on the idea that damaged areas should be rich in textural features compared with the same areas before damage or after reconstruction, which mainly consist of regular buildings showing fewer textural features (Liang, 2010; Liang et al., 2011). The widely used texture modeling is the grey level cooccurrence matrix (Haralick, 1973) due to its simplicity and low computational complexity, which has been proved very efficient in texture modeling. The textural feature inverse difference moment (indicating the local homogeneity of an image) is calculated within a $5 \times 5$ pixel window, which makes a good distinction between the original two images.

In this paper, the resulting chi-square image incorporating all the information of MAD variates is used for the final thresholding. This step is carried out using the ImageJ toolbox (Landini, 2010, ImageJ, 2011) providing different automatic thresholding methods. Here, the common global thresholding method by Otsu (1979) is applied. The algorithm searches for the optimal threshold that maximizes the separability of the resultant classes in grey levels assuming that the image is composed of only two classes: object and background.

Fig. 3 shows some intermediate results of applying the IR-MAD algorithm to the dataset shown in Fig. 1. The top two images are the first two variates of the algorithm. The IRMAD algorithm results in $n$ variates when applied to two images with $n$ channels each. The different variates show different changes and are in descending order (therefore only the first two are shown here). The chi-square image as combination of all variates is shown in the lower right corner. After the post-processing (thresholding and morphological operators) the image is overlayed again to the manual measurement. The distribution of green and blue areas is similar to the result of image differencing, whereas the red areas are strongly reduced. The manual verification will be much faster with this result.

\subsection{D Change Detection}

A more sophisticated damage assessment analysis is provided using additionally 3D change detection approaches. Unfortunately for most catastrophic events no height information like DSM or just very high resolution satellite stereo image pairs is available for the pre-disaster time. So these approaches are normally only applicable in reconstruction applications. For monitoring issues VHR satellite stereo images are acquired following the crisis. 3D change detection methods show changes, which can hardly or even not be seen in comparing 2D imagery like changes in heights and volume of buildings, waste dumps, and other objects. 



Fig. 3: Same detail as Fig. 1. (Top) IR-MAD variate 1 and 2, White = change; (Bottom left) Result of MAD algorithm: chi-square image, lighter = higher probability of change; (Bottom right) Overlay of resulting IR-MAD binary map and manual measurement (legend: see Fig. 2)

As for both of the Haiti datasets stereo scenes were ordered, the DEM were already generated during the pre-processing (see above). Here, the DEM are used for change detection. The height differences are calculated by simple subtraction of both DEM. The resulting image can be seen in Fig. 4 (bottom). Here, another area of Port-au-Prince is choosen due to the blunders in the DEM as described above (no blunders in this area). The difference image shows clearly positive (white) and negative (dark) changes. Negative changes indicate a lower height in the second image, there some object has been removed. Positive changes indicate some increase in height. As can be clearly seen in the upper left corner, the formerly empty space has been filled with refugee tents and even some building as indicated in the DEM change map. Also in the lower part of the image, some building has been extended, which cannot be seen in the 2D images.

Next step in the 3D change detection would be the automatic post-processing of the 3D difference image as e.g. shown in (Tian et al. 2011 and Chaabouni et al, 2011). Some threshold has to be defined to differentiate between measurement inaccuracy and real height changes. Additionally, some improvemtents of the DEM generation algorithm is needed in densly populated areas.

\section{Conclusions and Outlook}

The article shows first results of the application of the service chains for damage assessment and reconstruction monitoring developed in the context of G-MOSAIC. The results of the different change detection algorithms received with the Haiti datasets are 
compared to manual measurements retrieved also from these datasets. Further analysis is necessary, e.g. the comparison to the PDNA database for independent results. However, as it was already experienced during the rapid mapping, only a fraction of the damages can be seen in the VHR satellite data in comparison to the airborne data. But it can also be stated that the main areas of change are detected and give substantial hints for further manual evaluations. Therfore semi-automatic evaluation will be the method of choice for satellite data analysis in this context.
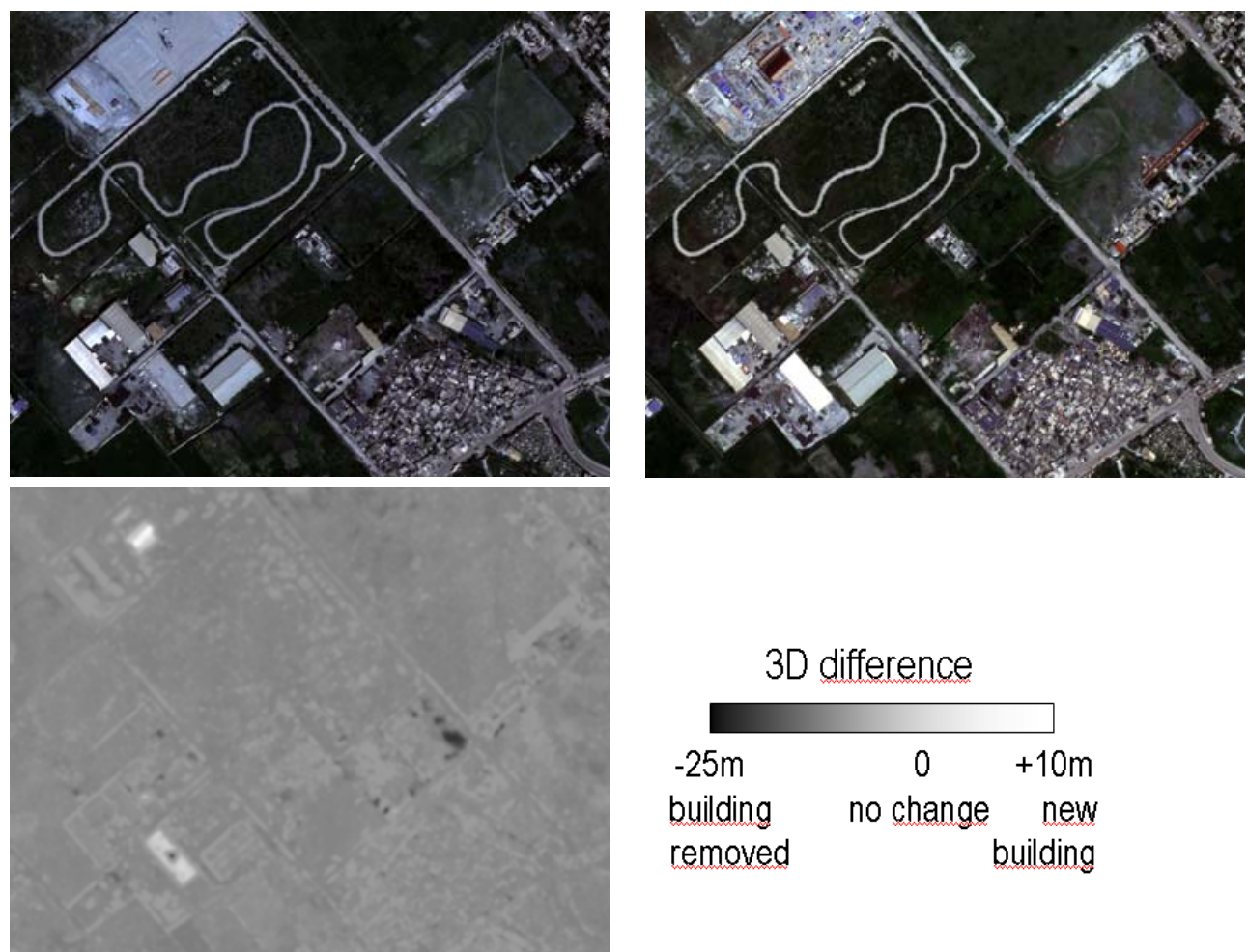

Fig. 4: (Top) Subset $(650 \mathrm{~m} \times 500 \mathrm{~m})$ of Port-au-Prince, Haiti showing the development of another refugee camp after the earthquake on 2010/01/12 (upper left corner). (Left) GeoEye, 2010/01/16; (Right) GeoEye 2010/08/18; (Bottom) Difference of DEM generated from the two stereo images

The various methods are in different development stages with the object to get a (semi-) automatic algorithm. Image differencing and IR-MAD incorporating texture information can already be applied completely automatically and only a manual verification of the results is necessary afterwards. Besides the qualitative analysis given above, Tab. 1 shows some quantitative statistical measures of the results. Completeness varies around $50 \%$, so only half of the changes are detected. But as can be seen in the figures above, no changed area remains completely undetected. The correctness is very low for the changes before/after the earthquake ( $9 \%$ or $19 \%$ ), whereas the changes occuring in the half year after the earthquake can be detected better. The IR-MAD algorithm incorporating texture information has a very promising value of $89 \%$ correctness. All these figures also show that the IR-MAD algorithm usually performs better (or at least similar, but never worse) than the image differencing.

$3 \mathrm{D}$ change detection is not yet compared to some reference data (and there are only 2D reference data available at all), but it is shown that changes can be detected. It results in the identification of changes, which can hardly or even not be seen in comparing 2D imagery like changes in heights and volume of buildings or other objects. Unfortunately for most catastrophic events no height information like DSM or just very high resolution satellite stereo image pairs are available for the pre-disaster time, but this might change in future. The intention of this research of change detection methods is that the developed methods (the best one or a combination of various) can be used in a semi-automatic way for future disaster monitoring applications where no airborne data are available. 
Tab. 1: Statistical measures for two change detection algorithms (Image differencing and IR-MAD incorporating texture information) and two time frames each (PRE=2009/10/01 $\rightarrow$ PO1=2010/01/16

\begin{tabular}{|c|c|c|c|c|c|}
\hline \multirow{2}{*}{$\begin{array}{l}\text { Quality } \\
\text { measure }\end{array}$} & \multirow[t]{2}{*}{ Formula } & \multicolumn{2}{|c|}{ Image differencing } & \multicolumn{2}{|c|}{ IR-MAD with texture } \\
\hline & & $\begin{array}{l}\text { PRE } \rightarrow \\
\text { PO1 }\end{array}$ & $\begin{array}{l}\text { PO1 } \rightarrow \\
\text { PO2 }\end{array}$ & $\begin{array}{l}\text { PRE } \rightarrow \\
\text { PO1 }\end{array}$ & $\begin{array}{l}\text { PO1 } \rightarrow \\
\text { PO2 }\end{array}$ \\
\hline $\begin{array}{l}\text { No of pixels } \\
\text { C / C }\end{array}$ & $T P(\square)$ & 11091 & 75072 & 14352 & 77517 \\
\hline $\begin{array}{l}\text { No of pixels } \\
\text { C / no } C\end{array}$ & $F P(\square)$ & 111921 & 68487 & 59021 & 10104 \\
\hline $\begin{array}{l}\text { No of pixels } \\
\text { no } C \text { / C }\end{array}$ & $F N(\square)$ & 10975 & 81032 & 7714 & 78587 \\
\hline $\begin{array}{l}\text { No of pixels } \\
\text { no } \mathrm{C} / \text { no } \mathrm{C}\end{array}$ & $T N(\boldsymbol{\square})$ & 866013 & 775409 & 918913 & 833792 \\
\hline $\begin{array}{l}\text { Completeness } \\
\text { (\%) }\end{array}$ & $\frac{100 \cdot T P}{T P+F N}$ & 50.3 & 48.1 & 65.0 & 49.7 \\
\hline $\begin{array}{l}\text { Correctness } \\
(\%)\end{array}$ & $\frac{100 \cdot T P}{T P+F P}$ & 9.0 & 52.3 & 19.6 & 88.5 \\
\hline $\begin{array}{l}\text { Quality } \\
\text { Percentage } \\
\text { (\%) }\end{array}$ & $\frac{100 \cdot T P}{T P+F P+F N}$ & 8.3 & 33.4 & 17.7 & 46.6 \\
\hline $\begin{array}{l}\text { Overall } \\
\text { Accuracy (\%) }\end{array}$ & $\frac{100 \cdot(T P+T N)}{T P+F P+F N+T N}$ & 87.7 & 85.0 & 93.3 & 91.1 \\
\hline
\end{tabular}

\section{Acknowledgement}

The authors acknowledge the help during processing with different parameters and the manual measurement of the test datasets carried out by Hans-Joachim Schneider and Anne de la Borderie.

\section{References}

BOVOLO, F. \& BRUZZONE, L., 2007: Image Information Mining in Time Series (IIM-TS): Applicable Algorithms and Methods. - Project Report.

CHAABOUNI-CHOUAYAKH, H., D'ANGELO, P., KAUSS, T., REINARTZ, P., 2011: Automatic Urban Area Monitoring Using Digital Surface Models and Shape Features. - Joint Urban Remote Sensing Event, Munich, Germany, 11-13 April 2011.

D’ANGELO, P., SCHWIND, P., KRAUSS, T., BARNER, F. \& REINARTZ, P., 2010: Automated DSM based Georeferencing of CARTOSAT-1 Stereo Scenes. - The International Archives of the Photogrammetry, Remote Sensing and Spatial Information Sciences, Volume XXXVIII-14-7/W5.

GROHMAN, G., KROENUNG, G. \& STREBECK, J., 2006: Fillling SRTM voids: The Delta Surface Fill Model. - Photogrammetric Engineering and Remote Sensing 72 (3): 213-216.

HARALICK, R.M., 1973: Textural Features for Image Classification. - IEEE Transactions on System, Man and Cybernetics, 3 (6): 610-621.

ImageJ Toolbox, 2011: Image Processing and Analysis in Java. http://rsb.info.nih.gov/ij/, Accessed January 2011.

LANDINI, G., 2010: Auto Threshold and Auto Local Threshold. http://www.dentistry.bham.ac.uk/ landinig/software/autothreshold/autothreshold.html, Accessed January 2011.

LIANG, W., 2010: Change Detection for Reconstruction Monitoring using Very High Resolution Optical Data. - Master’s thesis, TU München, 71 pages. 
LIANG, W., HOJA, D., SCHMITT, M. \& STILLA, U., 2011: Comparative Study of Change Detection for Reconstruction Monitoring based on Very High Resolution Optical Data. Joint Urban Remote Sensing Event, Munich, Germany, 11-13 April 2011.

MÜLLER, R., KRAUSS, T., SCHNEIDER, M. \& REINARTZ, P., 2010: A Method for Geometric Processing of Optical Satellite Images Using Automatically Determined Ground Control Information. - The International Archives of the Photogrammetry, Remote Sensing and Spatial Information Sciences, Volume XXXVIII part 1.

NIELSEN, A.A., 2007: The Regularized Iteratively Reweighted MAD Method for Change Detection in Multi- and Hyperspectral Data. - IEEE Transactions on Image Processing, 16(2): 463-478.

OTSU, N., 1979: A Threshold Selection Method from Gray-Level Histograms. - IEEE Transactions on System, Man and Cybernetics, 9(1): 62-66.

SINGH, A., 1989: Digital Change Detection Techniques using Remotely-sensed Data. - International Journal of Remote Sensing, 10 (6): 989-1003.

TIAN, J., CHAABOUNI-CHOUAYAKH, H., REINARTZ, P., 2011: 3D Building Change Detection from High Resolution Spaceborne Stereo Imagery. - International Workshop on Multiplatform/multi-sensor Remote Sensing and Mapping, IEEE Xplore, Xiamen, China. 